\title{
The comparison of dose distribution of different $3 D$ conformal and conventional radiotherapy plans for gastric cancer
}

\author{
M. Izmirli*, G. Yilmazer, T. Cakir, Z.A. Palabiyik, M. Nart
}

Yuzuncu Yll University, School of Medicine, Department of Radiation Oncology, 65080/Van, Turkey

\begin{abstract}
- Original article

\author{
*Corresponding author: \\ Dr. Mustafa Izmirli, \\ Fax: +905352583206 \\ E-mail: \\ izmirlimustafa@hotmail.com
}

Revised: Sept. 2015

Accepted: Nov. 2015

Int. J. Radiat. Res., July 2016; 14(3): 197-203

DOI: 10.18869 /acadpub.ijrr.14.3.197

Aims: It was aimed to investigate postoperative conformal radiotherapy planning that provides the best target volume and the least dose for critical organs in cancers of stomach. Methods: This study was conducted on the CT simulation images of thirty patients diagnosed with gastric cancer. Target volumes and the organs at risk were contoured. AP-PA reciprocal parallel field conventional plan and three- and four-field 3D conformal plans were created using linear accelerator. Target volumes and doses consumed by organs at risk were compared by dose-volume histograms. Results: While a sufficient dose could be applied to target volumes in all plans with conformal planning, average figures showed that $95 \%$ of porta hepatis area failed to take the prescribed dose (D95) in some plans by using AP-PA reciprocal parallel zone conventional plans. The most convenient protection for spinal cord, heart and kidneys was obtained by conformal four-field technique and the liver doses were increased in conformal four-field plans but did not exceed the tolerance dose. And also, in the conventional AP-PA reciprocal parallel field plans, tolerance dose of spinal cord (4500 cGy) was exceeded. Conclusion: In this study, conformal four-field technique was superior considering target volume dose distributions, and especially spinal cord doses in all localizations and heart doses in cardia tumors. Kidney doses were also reduced in conformal four-field planning, but failed to reach statistical significance. There was a not exceeding tolerance limits dose increase in liver.
\end{abstract}

Keywords: Gastric cancer, 3-dimensional conformal radiotherapy, dose-volume histogram.

\section{INTRODUCTION}

Gastric cancer is a malignancy with aggressive course and bad prognosis (1). It was the fourth most common cancer $(934,000$ new cases) and the second most common cause of death from cancer $(700,000$ deaths) all over the world in 2002, especially in developing countries (2). Intergroup Study (INT-0116) showed that chemoradiotherapy after surgery had beneficial effects on disease-free survival and general survival $(3,4)$.

The aim of radiotherapy is to apply optimum planned dose to tumor cells while protecting surrounding normal tissues as much as possible
(5). Modern imaging methods such as CT, MRI and PET-CT enable radiation oncologist to obtain three-dimensional (3D) anatomic and functional images of patient, and to define the tumor and surrounding normal tissues better (6). The aim of 3D conformal radiotherapy(3DCRT) is to adjust the radiation dose to target volume while exposing the surrounding normal tissues with lower doses (6).

In this study, we aimed to obtain the best dose distribution to target volumes by 3DCRT and to make the most convenient plan protecting organs at risk at the highest level in gastric cancer patient who undergo chemoradiotherapy after surgery. This study included 30 patients 
with gastric cancer located in cardia, corpus or antrum regions. Contours of target fields and criticalorgans were revised after CT simulation. The most convenient treatment plan was investigated by comparing dose-volume histogram results of AP-PA reciprocal parallel field conventional plan for linear accelerator and three- and four-field conformal plans.

\section{MATERIALS AND METHODS}

This study was conducted on CT simulation images of 30 patients with gastric cancer (10 cardia tumor, 10 corpus tumor and 10 antral tumor) who were diagnosed between January 2008 and December 2009 in Radiation Oncology Clinics, Yuzuncu Yil University Faculty of Medicine. This study approved by "Yuzuncu Yil University Faculty of Medicine Clinical Research Ethical Committee". Of the patients, 7 were female and 23 were male with the mean age of 59 (range 37-82 years). Patients had stage IIB-IIIC (AJCC 2010) adenocarcinoma. 21 patients had total gastrectomy, and 9 patients had subtotal gastrectomy. Patient anteriorposterior median thickness was $21.2 \mathrm{~cm}$ (range $18.3-26.8 \mathrm{~cm}$ ) in simulation tomography.

CT images (Siemens Somatom) taken by 5 mm section intervals at treatment position were transferred to a 3D planning system (CMS XIO Release 4.34.02). Contouring was performed by the same investigator for each section. Spinal cord, heart, liver, right and left kidneys were contoured as the organs at risk. Patients were divided into 3 groups: Group I, patients with cardia tumor; Group II, patients with corpus tumor; Group III, patients with antrum tumor. The target volumes were contoured according to the different involvement regions of stomach, the study of Tepper and Gunderson (7), and NCCN Guidelines (8). The remaining stomach was evaluated according to the pathological data. Subjects with a T4 tumor or a positive lymph node were contoured while subjects with T2-3 tumors and wide surgical border $(>5 \mathrm{~cm})$ were not contoured. Tumor bed was contoured in all T3-4 tumors. Lines of proximal and distal anastomoses were contoured in all patients.
Contouring studies of the groups were performed as defined below;

Group I: Adjacent paraesophageal, perigastric, celiac, splenic hilum, suprapancreatic \pm pancreaticoduodenal and porta hepatic lymph nodes \pm in case of sufficient lymph node dissection, N0 had no contouring, N1-2 had contouring), distal $3-5 \mathrm{~cm}$ part of esophagus, medial left hemidiaphragm as the tumor bed and adjacent pancreatic corpus were contoured.

Group II: Perigastric, celiac, splenic hilum, suprapancreatic, pancreaticoduodenal, porta hepatic lymph nodes and pancreas corpus and tail as the tumor bed were contoured.

Group III: Perigastric, pancreaticoduodenal, porta hepatic, celiac, suprapancreatic \pm splenic hilum $( \pm$ in case of sufficient lymph node dissection, N0 had no contouring, N1-2 had contouring) and pancreas head, corpus and duodenum 1-2. Parts or a $3-5 \mathrm{~cm}$ margin of duodenal stump as the tumor bed were contoured.

In all treatment plans of this study, daily 1.8 Gy with 25 fraction and total 45 Gy radiotherapy dose was planned for linear accelerator (Siemens Oncor 10MV). Organs at risk were protected with Multileaf collimators (MLCs) for each treatment plan techniques.

Plan I: The conventional plan with classical AP-PA reciprocal parallel field use, midline dosing of equal weight, and the protection of organs at risk (Conventional plan).

Plan II: The conformal plan including all target volumes besides critical organ protection. Anterior (35\% weight) - posterior (35\% weight) - left side $(30 \%$ weight $)$ field conformal plan (Conformal plan three-field).

Plan III: The conformal plan including all target volumes besides critical organ protection. Anterior (30\% weight) - posterior (30\% weight) - left side (25\% weight) - right side (15\% weight) field conformal plan (Conformal plan four- field) (figure 1).

Volumes and dose limits for organs at risk were determined by NCCN guidelines and EORTC-ROG expert opinion (8,9). Thus, maximal spinal cord dose should not exceed 45 Gy $(8,9)$. For heart, the volume taking 40 Gy dose should be less than $30 \%$ of whole heart volume (V40<30\%), and the volume taking 25 Gy dose 
should be less than $50 \%$ of whole heart volume (V25<50\%)(9). For liver, the volume taking $30 \mathrm{~Gy}$ dose should be less than $30 \%$ of whole liver volume $(\mathrm{V} 30<30 \%)^{(8,9)}$. For kidneys, the volume taking 20 Gy dose should be less than $70 \%$ of whole kidney volume (V20<70\%) and the contralateral kidney volume taking 20 Gy dose should be less than $30 \%$ of whole kidney volume $(\mathrm{V} 20<30 \%)^{(9)}$.

For each treatment plan, dose-volume histograms were used and the value of volume percent that take the calculated dose for target volume (V45), maximal dose (cGy) values for spinal cord, and values of V25-V40 for heart, V30 for liver, V20 for right and left kidney (\%volume) were recorded.

Statistical analyses included repeated measures ANOVA and Tukey's multiple comparisons tests. Statistical significance level was adjusted to 5\% and SPSS 15 statistical software program was used for all calculations.

\section{RESULTS}

\section{Evaluation for target volumes}

In conformal approach, following the administration of prescription dose (45 Gy), the
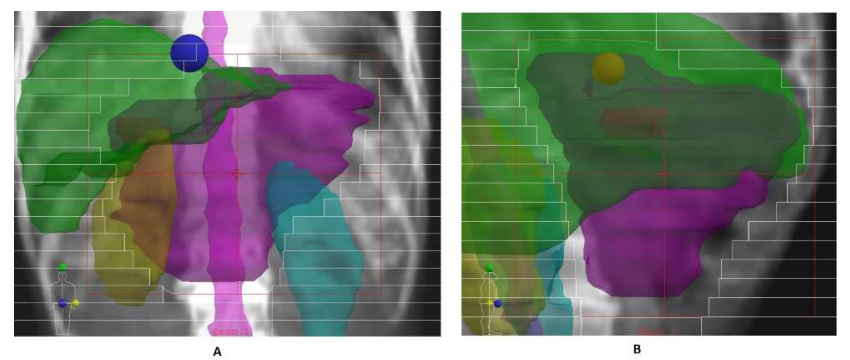

Figure 1. Conformal plan four-field AP-PA (A), right side (B) DRR images and contours; PTV (purple), porta hepatis (red), liver (green), right kidney (yellow), left kidney (cyan). doses received by at least $95 \%$ of the planned target volumes (PTV) (D95) were examined in all plans. In evaluation of D95 values in conventional plans, other targets obtained 95\% and higher doses while porta hepatis lymph nodes region remained lower than the prescribed dose in some plans (figure 2 and 3 ). In conventional plan of corpus tumors, the dose of porta hepatis lymph nodes region $(65.9 \pm 45.3 \%$, range $0-100 \%)$ reached to statistical significance when compared to four-field conformal plan. It failed to reach statistical significance level in antrum tumors (77.2 \pm 40.1 , range $0.3-100 \%$ ) (table 1 ).

\section{Evaluation for the organs at risk}

Maximal doses were compared in the evaluation of spinal cord between three groups (groups 1, 2 and 3) and the plans (three-field conformal, four-field conformal and conventional) (table 2). No statistical differences were found in inter-group comparisons. In the inter-plan comparisons, the lowest dose value was obtained in the conformal plan four-field and the differences from other plans were significant (Group I / II / III; 3533.8 \pm 255.3 cGy / $3485 \pm 321.7$ cGy / 3516 \pm 263.4 cGy respectively). In the conventional plan, the dose of spinal cord was higher than the tolerance limits (Group I / II / III; 4943.6 \pm 111.0 cGy / 4906.5 \pm 78.4 cGy / 4935.1 \pm 103.9 cGy respectively) (figure 4C: Representative DVHs for the comparison between the plans for spinal cord).

We compared the mean V25 and V40 percent values of heartbetween the groups and the plans (table 3). Inter-group comparisons showed that V25 (Plan I / II / III; 48.0 \pm 18.5 / 45.9\% \pm 17.9 / $48.3 \% \pm 19.2$ respectively) and V40 (Plan I / II /
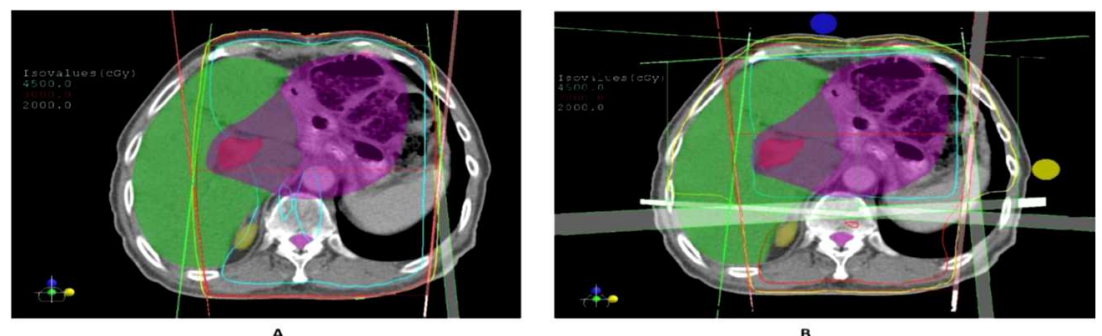

B

Figure 2. The dose decrease in porta hepatis in the plan in which conventional AP-PA reciprocal parallel dose was defined in the midline (A). Four field conformal planning made by using linear accelerator $10 \mathrm{MV}$ energy (B). Cyan line 4500, red line 3000, yellow line $2000 \mathrm{cGy}$ isodose curve; solid red is porta hepatis. 
III; $21.7 \% \pm 6.8$ / $16.3 \% \pm 5.2$ / $38.5 \% \pm 20.5$ respectively) values of cardia tumor were significantly higher than those at the other localizations in all plans. In inter-plan comparisons, conformal plan four-field showed the lowest V25 and V40 values with respect to the other plans. Only corpus tumors reached statistical significance in V25 values. V40 values in cardia and corpus tumors were significantly lower in conformal plan four-field than those in the other plans.

Liver V30 values were also compared between the study groups and between the different plans (table 3). Inter-group comparisons showed no difference. Inter-plan comparisons revealed that conformal plan four-field had significantly higher values than the other plans (Group I / II / III; 27.0\% \pm 4.4 / $27.5 \% \pm 7.1 / 29.0 \% \pm 6.5$ respectively), but these values did not exceed the tolerance dose (V30<30\%) (figure 4D: Representative DVHs for the comparison between the plans for liver).

V20 values were compared between the groups and plans for right kidney (table 3). Inter -group comparisons showed no difference. Inter-plan comparisons showed that conformal plan four-field had lower values than the other plans, and the difference was significant in
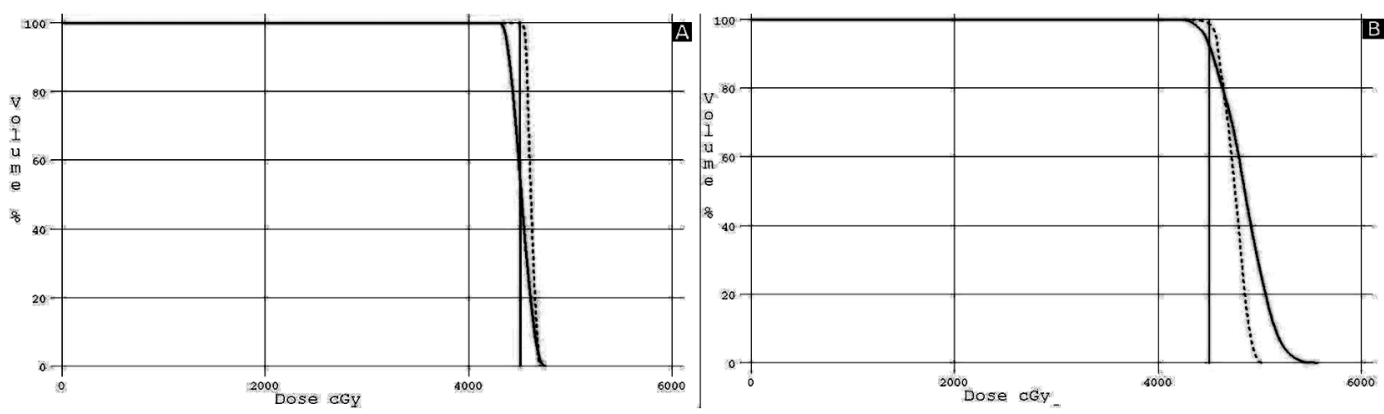

Figure 3. DVH image comparisons of porta hepatis coverage (A) and PTV coverage (B) in conventional AP-PA reciprocal parallel plan and four field conformal plan; cursor was at the dose of $4500 \mathrm{cGy}$, solid lines showed conventional AP-PA reciprocal parallel plan and dotted lines showed four field conformal plan. Porta Hepatis V45 \%54.97 and PTV45 \%92.27 in conventional AP-PA reciprocal parallel plan; Porta Hepatis V45 \%100 and PTV45 \%99.0 in four field conformal plan.
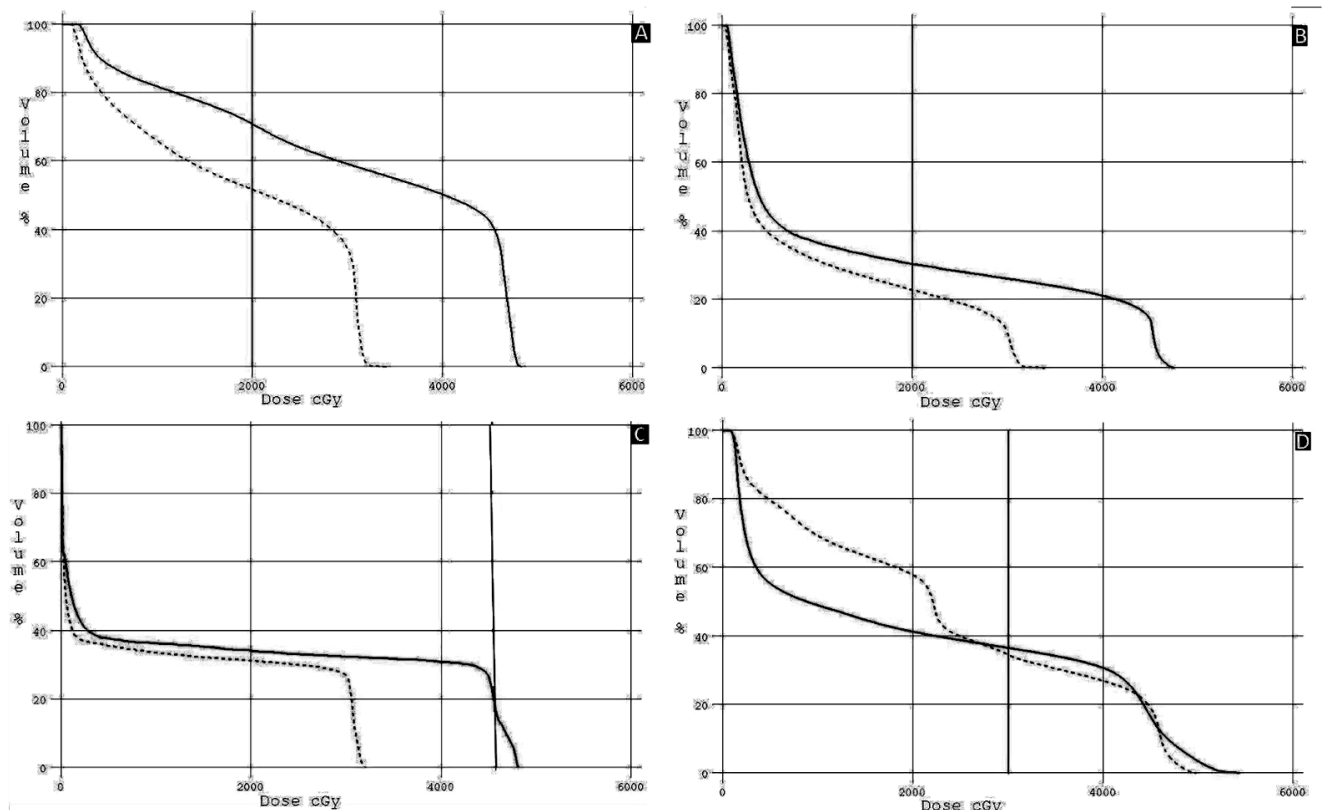

Figure 4. DVH image comparisons of right kidney; cursor was at the dose of $2000 \mathrm{cGy}(\mathrm{A})$, left kidney; cursor was at the dose of 2000 cGy (B), spinal cord; cursor was at the dose of 4500 cGy (C) and liver; cursor was at the dose of 3000 cGy (D) in conventional AP-PA reciprocal parallel plan and four field conformal plan; solid lines showed conventional AP-PA reciprocal parallel plan and dotted lines showed four field conformal plan. 
cardia and antrum tumors (Group I / II / III; $10.6 \% \pm 6.6$ / $15.7 \% \pm 10.5$ / $16.1 \% \pm 9.3$ respectively). V20 values remained within the tolerance limits (V20<30\%). V20 values were compared for left kidney between three groups and the plans used in the study. Inter-group comparisons showed no difference. Inter-plan comparisons showed that conformal plan four-field (Group I / II / III; 35.3\% \pm 13.9 / 42.0\% $\pm 13.3 / 43.0 \% \pm 10.9$ respectively) had significant difference in corpus tumors comparing with the conventional plan. Nevertheless, V20 values did not exceed tolerance doses in all plans (V20<70\%). (figure $4 \mathrm{~A}$ and B: Representative DVHs for the comparison between the plans for right and left kidney).

Table 1. Comparison of D95 values (Volume percent of at least 45 Gy dose exposition) of the dose taken by porta hepatis. Porta hepatis was excluded from analysis as it was not included CTV in all patients with cardia tumor.

\begin{tabular}{|c|c|c|c|c|}
\hline & \multicolumn{2}{|c|}{ Group 2 (Corpus) } & \multicolumn{2}{c|}{ Group 3 (Antrum) } \\
\hline & Mean \% & St. Dev. & Mean \% & St. Dev. \\
\hline Three field conformal & $99,9 \mathrm{~A} \mathrm{ab}$ &, 4 & $99,8 \mathrm{~A} \mathrm{a}$ &, 5 \\
\hline Four field conformal & $100,0 \mathrm{~A} \mathrm{a}$ &, 0 & $100,0 \mathrm{~A} \mathrm{a}$ &, 0 \\
\hline Conventional & $65,9 \mathrm{~A} \mathrm{~b}$ & 45,3 & $77,2 \mathrm{~A} \mathrm{a}$ & 40,1 \\
\hline
\end{tabular}

A, B $\rightarrow$ Difference between the means that labelled with a different upper case in the same line is significant $(p<0.05)$. $a, b \downarrow$ Difference between the means that labelled with a different lower case in the same column is significant ( $p<0.05)$.

Table 2. Descriptive statistics and comparisons of the means of maximal doses (cGy) for spinal cord.

\begin{tabular}{|c|c|c|c|c|c|c|}
\hline & \multicolumn{2}{|c|}{ Group 1 (Cardia) } & \multicolumn{2}{c|}{ Group 2 (Corpus) } & \multicolumn{2}{c|}{ Group 3 (Antrum) } \\
\hline & Mean & St. Dev. & Mean & St. Dev. & Mean & St. Dev. \\
\hline Three field conformal & $4376,6 \mathrm{~A} \mathrm{~b}$ & 289,5 & $4279,1 \mathrm{~A} \mathrm{~b}$ & 275,6 & $4395,4 \mathrm{~A} \mathrm{~b}$ & 192,6 \\
\hline Four field conformal & $3533,8 \mathrm{~A} \mathrm{c}$ & 255,3 & $3485,0 \mathrm{~A} \mathrm{c}$ & 321,7 & $3516,4 \mathrm{Ac}$ & 263,4 \\
\hline Conventional & $4943,6 \mathrm{~A} \mathrm{a}$ & 111,0 & $4906,5 \mathrm{~A} \mathrm{a}$ & 78,4 & $4935,1 \mathrm{Aa}$ & 103,9 \\
\hline
\end{tabular}

A, B $\rightarrow$ Difference between the means that labelled with a different upper case in the same line is significant $(p<0.05)$.

$a, b \downarrow$ Difference between the means that labelled with a different lower case in the same column is significant $(p<0.05)$.

Table 3. Descriptive statistics and a comparison of the results between percent volumes of heart, liver and kidneys regarding with different treatment plans in patients with gastric cancer.

\begin{tabular}{|c|c|c|c|c|c|c|c|}
\hline & & \multicolumn{2}{|c|}{ Group 1 (Cardia) } & \multicolumn{2}{|c|}{ Group 2 (Corpus) } & \multicolumn{2}{|c|}{ Group 3 (Antrum) } \\
\hline & & Mean & St. Dev. & Mean & St. Dev. & Mean & St. Dev. \\
\hline \multirow{3}{*}{$\begin{array}{l}\text { Heart } \\
\text { V25 }\end{array}$} & Three field conformal & $48,0 \mathrm{~A} \mathrm{a}$ & 18,5 & $29,1 \mathrm{~B} \mathrm{a}$ & 9,2 & $22,1 \mathrm{~B} \mathrm{a}$ & 10,5 \\
\hline & Four field conformal & $45,9 \mathrm{~A} \mathrm{a}$ & 17,9 & $27,2 \mathrm{~B} \mathrm{~b}$ & 9,1 & $21,0 \mathrm{~B}$ a & 8,1 \\
\hline & Conventional & $48,3 \mathrm{~A} \mathrm{a}$ & 19,2 & $29,6 \mathrm{~B} \mathrm{a}$ & 9,4 & $24,0 \mathrm{~B} \mathrm{a}$ & 11,2 \\
\hline \multirow{3}{*}{$\begin{array}{l}\text { Heart } \\
\text { V40 }\end{array}$} & Three field conformal & $21,7 \mathrm{~A} \mathrm{~b}$ & 6,8 & $14,8 \mathrm{~B} \mathrm{~b}$ & 7,4 & $11,1 \mathrm{~B} \mathrm{a}$ & 7,0 \\
\hline & Four field conformal & $16,3 \mathrm{Ac}$ & 5,2 & $9,8 \mathrm{~B} \mathrm{C}$ & 4,7 & $9,6 \mathrm{~B} \mathrm{a}$ & 6,1 \\
\hline & Conventional & $38,5 \mathrm{~A} \mathrm{a}$ & 20,5 & $20,4 \mathrm{~B} \mathrm{a}$ & 7,8 & $15,5 \mathrm{~B} \mathrm{a}$ & 9,1 \\
\hline \multirow{3}{*}{$\begin{array}{l}\text { Liver } \\
\text { V30 }\end{array}$} & Three field conformal & $25,8 \mathrm{~A} \mathrm{~b}$ & 4,0 & $28,4 \mathrm{~A} \mathrm{a}$ & 4,3 & $27,7 \mathrm{~A} \mathrm{~b}$ & 6,6 \\
\hline & Four field conformal & $27,0 \mathrm{~A} a$ & 4,4 & $27,5 \mathrm{Aab}$ & 7,1 & $29,0 \mathrm{~A} a$ & 6,5 \\
\hline & Conventional & $25,1 \mathrm{~A} \mathrm{~b}$ & 3,8 & $26,5 \mathrm{~A} \mathrm{~b}$ & 4,0 & $25,4 \mathrm{~A} \mathrm{~b}$ & 8,5 \\
\hline \multirow{3}{*}{$\begin{array}{c}\text { Right } \\
\text { Kidney } \\
\text { V20 }\end{array}$} & Three field conformal & $11,3 \mathrm{Aab}$ & 6,5 & $17,9 \mathrm{~A} \mathrm{a}$ & 11,7 & $18,0 \mathrm{~A} \mathrm{~b}$ & 10,5 \\
\hline & Four field conformal & $10,6 \mathrm{~A} \mathrm{~b}$ & 6,6 & $15,7 \mathrm{~A} \mathrm{a}$ & 10,5 & $16,1 \mathrm{~A} \mathrm{C}$ & 9,3 \\
\hline & Conventional & $11,9 \mathrm{~A} \mathrm{a}$ & 6,6 & $18,3 \mathrm{~A} \mathrm{a}$ & 11,7 & $19,2 \mathrm{~A}$ a & 11,6 \\
\hline \multirow{3}{*}{$\begin{array}{c}\text { Left } \\
\text { Kidney } \\
\text { V20 }\end{array}$} & Three field conformal & $35,7 \mathrm{~A} \mathrm{a}$ & 16,8 & $42,0 \mathrm{~A} \mathrm{~b}$ & 13,8 & $42,8 \mathrm{~A}$ a & 10,9 \\
\hline & Four field conformal & $35,3 \mathrm{~A} \mathrm{a}$ & 13,9 & $42,0 \mathrm{~A} \mathrm{~b}$ & 13,3 & $43,0 \mathrm{~A} a$ & 10,9 \\
\hline & Conventional & $35,2 \mathrm{~A} \mathrm{a}$ & 17,1 & $44,4 \mathrm{~A} \mathrm{a}$ & 14,0 & $43,3 \mathrm{~A} a$ & 10,6 \\
\hline
\end{tabular}

A, B $(\rightarrow)$ : The difference between the means that labelled with a different upper case in the same line is significant $(p<0.05)$.

$a, b, c(\downarrow)$ : The difference between the means that labelled with a different lower case in the same column is significant $(p<0.05)$.

$\mathrm{Vx}$ : percent volume of an organ that took at least ' $\mathrm{x}$ ' Gy dose.

(Heart: a comparison of the means of percent volume took V25-V40, 25 Gy and 40 Gy in DVH).

(Liver: a comparison of the means of percent volume took V30, $30 \mathrm{~Gy}$ in DVH). 


\section{DISCUSSION}

In this study, the doses of postoperative AP-PA conventional plan, conformal plan three-field and conformal plan four-field were compared in 30 patients with gastric adenocarcinoma. In target volume evaluation, conformal plans obtained the estimated doses in all tumor localizations. Evaluation of conventional AP-PA plans revealed that the dose of porta hepatis lymph nodes was lower than the estimated dose in some plans, and the difference was significant in corpus tumors. In the evaluation of spinal cord dose, the dose of AP-PA conventional plan was significantly higher and it was above the tolerance dose (4500 cGy). V25 and V40 percent values of heart were significantly higher in all plans of cardia tumors. Although conformal plan four-field was low in all plans, only V25 values in corpus tumors and V40 values in cardia and corpus tumors reached statistical significance. Liver V30 percent values in conformal plan four-field were significantly higher than those in the other plans but the tolerance doses were not exceeded. Although right and left kidney V20 results were within the tolerance limits in all plans, conformal plan four-field obtained lower values when compared to the other plans. This difference was significant for the right kidney in cardia and antrum tumors, and for the left kidney in corpus tumors.

Intergroup study (INT-0116) showed the beneficial effects of adjuvant chemoradiotherapy (CRT) on disease-free survival and general (overall) survival in gastric and gastroesophageal cancers, but $32 \%$ of the patients developed G3-4 toxicity, and $17 \%$ of the patients have had to stop treatment due to toxicity $(3,4)$. In that study, radiotherapy was applied by AP/PA conventional method.

In the retrospective study of Henning and coworkers, postoperative radiotherapy was evaluated in 63 patients with gastric cancer. Irradiation with four- or more fields resulted in significant reduction in grade 4-5 toxicity when compared to two-field irradiation. They suggested that, in combined model treatments, use of more than two irradiation field might reduce toxicity ${ }^{(10)}$.

EORTC-ROG expert opinion stated that 3D conformal planning and use of dose-volume histograms are necessary in neoadjuvant radiotherapy volume and treatment planning study in gastric and gastroesophageal junction adenocarcinoma. $95 \%$ of the PTV should be covered, and the whole volume should not take a dose lower than $95 \%$ of the prescribed dose (9).

NCCN Clinical Practical Oncology Guide recommends CT simulation and 3D planning in radiotherapy planning of gastric cancers. Furthermore, four-field technique (AP/PA and opposed lateral) can be obtained sparing spinal cord with improved dose homogeneity (8).

Tepper and Gunderson suggested that primary tumors arising from different regions of stomach may show different growth and progression patterns, and field organization should be performed in adjuvant radiotherapy (7).

Caudry et al. (11) suggested that CTV should be based on 3 factors including tumor bed volume, peritoneal volume and lymphatic volume. Leong et al. (12) compared 3DCRT and AP-PA conventional technique in gastric cancer. They provided a better dose with conformal planning which received $99 \%$ of the PTV dose when compared to AP/PA technique (93\%). They demonstrated lower radiation doses for right and left kidneys, and spinal cord. The liver dose was higher but remained below the tolerance dose (12).

Marcenaro et al. (13) compared 3D multi-planar conformal technique and standard 2-field technique in postoperative radiotherapy of patients with gastric cancer. The dose reduced in both kidneys and the reduction in the right kidney dose was greater, although the liver dose increased (13). In addition to achievement of reductions in spinal cord dose and kidney dose in the above-mentioned studies, our study provided significant reduction in the heart dose V25-V40 values with conformal four-plan in patients with a cardia tumor. 
In conclusion, conformal four-field planning provided a better dose distribution, sufficient amount to target volumes and the optimum protection for spinal cord and kidneys in all tumor localizations, and for heart especially in cardia tumors. Increases in liver dose did not exceed the tolerance dose. Due to lower radiation doses applied to normal tissues, reduced toxicity may be anticipated, but hepatic functions should be monitored as the liver doses-even if within the tolerance limits- may still increase.

\section{Conflict of interest: Declared None.}

\section{REFERENCES}

1. Yao JC, Mansfield PF, Pisters PWT, Feig BW, Janjan NA, Crane C, Ajani JA (2003) Combined-modality therapy for gastric cancer. Semin Surg Oncol, 21(4):223-7.

2. Parkin DM, Bray F, Ferlay J, Pisani P (2005) Global cancer statistics 2002. CA Cancer J Clin, 55(2):74-108.

3. Macdonald JS, Smalley SR, Benedetti J, HundahI SA, Estes NC, Stemmermann GN, et al. (2001) Chemoradiotherapy after surgery compared with surgery alone for adenocarcinoma of the stomach or gastroesophageal junction. $N$ Engl J Med, 345(10):725-30.

4. Macdonald JS, Smalley SR, Benedetti J, Estes N, Haller DG, Ajani JA, Gunderson LL, Jessup M, Martenson JA (2004) Preoperative combined radiation and chemotherapy improves disease-free survival (DFS) and overall survival (OS) in resected adenocarcinoma of the stomach and gastroesophageal junction: Undate of the results of Intergroup Study INT-0116 (SWOG 9008). Gastrointestinal Cancers Symposium Program / Proceedings, Abstract; $106 \mathrm{p}$.
5. Halperin E, Perez C, Brady L (2008) The discipline of radiation oncology. In: Perez and Brady's principles and practice of radiation oncology, 5th ed. (Halperin EC, Perez CA, Brady LW, eds) Lippincot Williams and Wilkins, Philadelphia, USA; 2-75 $\mathrm{p}$.

6. Purdy JA (2008) Three-dimensionel conformal radiotherapy; physics, treatment planning, and clinical aspects. In: Perez and Brady's principles and practice of radiation oncology. 5th ed. (Halperin EC, Perez CA, Brady LW, eds) Lippincot Williams and Wilkins, Philadelphia, USA; 218-38 p.

7. Tepper JE and Gunderson LL (2002) Radiation treatment parameters in the adjuvant postoperative therapy of gastric cancer.Semin Radiat Oncol, 12(2):187-95.

8. NCCN clinical practice guidelines in oncology (NCCN Guidelines) (2013) Gastric cancer (Including cancer in the proximal $5 \mathrm{~cm}$ of the stomach) Version 2.2013, NCCN.org. Principles of Radiation Therapy (GAST-F), 40-43 p.

9. Matzinger $O$, Gerber $E$, Bernstein $Z$, Maingon $P$, Haustermans K, Bosset JF et al. (2009) EORTC-ROG expert opinion: Radiotherapy volume andtreatment guidelines for neoadjuvant radiation of adenocarcinomas of the gastroesophageal junction and the stomach. Radiother Oncol, 92(2):164-75.

10. Henning GT, Schild SE, Stafford SL, Donohue JH, Burch PA, Haddock MG, et al. (2000) Results of irradiation or chemoirradiation following resection of gastric adenocarcinoma. Int J Radiat Oncol Biol Phys, 46 (3):589-98.

11. Caudry M, Ratoanina JL, Escarmant P, Maire JP (2001) Target volume in radiotherapy of gastric adenocarcinoma. Cancer Radiother, 5(5):523-33.

12. Leong $T$, Willis $D$, Joon DL, Condron $S$, Hui A, Ngan SY (2005) 3D conformal radiotherapy for gastric cancer-results of a comparative planning study. Radiother Oncol, 74(3):301-6.

13. Marcenaro M, Foppiano F, Durzu S, Barra S, Corvò R (2006) Kidney-sparing radiotherapy by multiple-field three-dimensional technique in the postoperative management of patients with gastric cancer: Comparison with standard two-field conformal technique. Tumori, 92(1):34-40. 
